\title{
Monitoring the ionospheric total electron content variations over the Korean Peninsula using a GPS network during geomagnetic storms
}

\author{
Byung-Kyu Choi ${ }^{1}$, Sang-Jeong Lee ${ }^{2}$, and Jong-Uk Park ${ }^{3}$ \\ ${ }^{1}$ Space Science Division, Korea Astronomy and Space Science Institute, Daejeon 305-348, South Korea \\ ${ }^{2}$ Department of Electronics Engineering, Chungnam National University, Daejeon 305-764, South Korea \\ ${ }^{3}$ Space Science Division, Korea Astronomy and Space Science Institute, Daejeon 305-348, South Korea
}

(Received December 10, 2010; Revised February 28, 2011; Accepted March 9, 2011; Online published September 7, 2011)

\begin{abstract}
We have established a regional ionospheric model (RIM) for investigating changes in the total electron content (TEC) over South Korea using 38 Korean GPS reference stations. The inverse distance weighted (IDW) interpolation method was applied to create a two-dimensional ionospheric map of vertical TEC units (TECU) based on a grid. To examine the diurnal patterns of ionospheric TEC over South Korea, we rst processed the GPS data from a geomagnetically quiet period of 10 days. In a second step, we compared the estimated GPSTEC variations with the changes in geomagnetic activity indices (the $K_{\mathrm{p}}$ and $D_{\mathrm{st}}$ indices) and the auroral electrojet index $\left(A_{\mathrm{E}}\right)$ as a function of universal time (UT) on 4 and 20 November, 2003. The GPS-TEC responses for those storm events were proportional to the geomagnetic activity at this mid-latitude location. The sudden increases in ionospheric TEC (SITEC) caused by the geomagnetic storms were detected. The variations in GPS-TEC may help reveal the processes of ionospheric disturbances caused by geomagnetic storms.
\end{abstract}

Key words: Regional ionospheric model, total electron content, inverse distance weighted interpolation, geomagnetic storm.

\section{Introduction}

The Global Positioning System (GPS) has been used as a valuable tool to monitor and estimate ionospheric total electron contents (TECs) (Lanyi and Roth, 1988; Mannucci et al., 1993; Sardon et al., 1994; Davis and Hartmann, 1997; Hernandez-Pajares et al., 1999). The TEC in the ionosphere is measured using two of the L band carrier frequencies, L1 at $1575.42 \mathrm{MHz}$ and L2 at $1227.60 \mathrm{MHz}$, because GPS signals are easily affected by the dispersive nature of the ionosphere. The propagation time of a signal is directly proportional to the integrated electron density along the signal path. By processing the data from a dual frequency GPS receiver, it is possible to calculate the number of free electrons that were encountered by the signal path from the satellite to the receiver. The magnitude of the TEC in the ionosphere is variable and depends on several factors such as season, geographical position, local time, the solar activity cycle, and geomagnetic storm occurrences (Fedrizzi et al., 2005; Jakowski et al., 2002; Immel et al., 2003; Tsurutani et al., 2004; Mannucci et al., 2005).

The responses of the ionosphere during storm events have been studied for several decades using numerous instruments, such as incoherent scatter radars (ISR) and ionosondes (Lei et al., 2004). However, ISRs are limited by their local coverage, and ionosondes can only observe the bottom part of the ionosphere, whereas ground-based GPS mea-

Copyright (C) The Society of Geomagnetism and Earth, Planetary and Space Sciences (SGEPSS); The Seismological Society of Japan; The Volcanological Society of Japan; The Geodetic Society of Japan; The Japanese Society for Planetary Sciences; TERRAPUB.

doi:10.5047/eps.2011.03.004 surements enable the ionosphere to be well monitored without several geometric limitations. Data from GPS networks enable us to measure the TEC of the ionosphere.

Many researchers have developed ionospheric models to derive GPS-TECs (Sardon et al., 1994). Recently, some researchers have used GPS network data to study ionospheric irregularities (Otsuka et al., 2002; Jin et al., 2004). Jakowski et al. (2002) suggested that TEC monitoring by GPS networks can contribute to research on space weather. Wan et al. (2005) investigated the sudden increase in total electron content (SITEC) caused by a very intensive solar are by using the observations from a worldwide GPS network. Zhao et al. (2005) investigated the response of the Equatorial Ionization Anomaly (EIA) to the superstorms of October-November 2003 using GPS data measured over a regional area. Tsugawa et al. (2006) also investigated medium-scale ionospheric disturbances that generally induce perturbations in ionospheric TECs by using the GPS Earth Observation Network (GEONET). The ground-based GPS networks available both globally and in regional areas also enable the assessment of variations in ionospheric TECs during both quiet and disturbed periods possible. The TEC derived from a GPS network is very useful in helping to understand upper atmosphere phenomena. When data from a ground-based GPS network is used, a map of the TEC over that region can be produced.

Early ionospheric modeling methods were mostly based on function- tting techniques such as those for spherical harmonics (Schaer, 1999), the broadcasting ionosphere model (Klobuchar, 1987) and polynomial functions. Gridbased ionospheric modeling has been extensively used for 
global and regional ionosphere recovery (Skone, 1998; Liao, 2000).

One of the largest solar X-ray flares occurred on 4 November 2003. The largest geomagnetic storm of solar cycle 23 occurred on 20 November 2003 due to a coronal mass ejection (CME). Extreme solar flares can result in various ionospheric phenomena: for example, a sudden increase of TEC (SITEC), storm-enhanced density (SED) and equatorial plasma bubbles (EPBs). The 28 and 29 October and 4 November, 2003, flares caused a TEC enhancement of about 30 percent (Tsurutani et al., 2006).

This paper presents a method for high-precision ionospheric TEC modeling using a local GPS network and an attempt to monitor the variation of the ionospheric TEC over the Korean Peninsula by using Korean GPS Network (KGN) data for 4 and 20 November, 2003. The vertical TEC maps obtained from densely-located local GPS receivers enable for a precise detection of the TEC response of the ionosphere. This ionospheric model is based on a two-dimensional grid. The inverse distance weighted (IDW) interpolation method is applied to generate the twodimensional ionospheric map of the vertical TECU. In order to monitor the TEC response over South Korea during storm periods, diurnal TEC patterns at any point for 10 quiet days are determined and the median TEC variation over the 10 days is also calculated. This data is used to monitor ionospheric TEC enhancements during storm periods. In addition, we have investigated the correlation between TEC responses and geomagnetic activity at mid-latitudes. We have also studied the GPS-TEC variation at the time of sudden storm commencement (SSC). The aim of this paper is to show and discuss some effects on the ionosphere TEC at mid-latitudes of the geomagnetic storms that occurred in November 2003.

\section{Korean GPS Network (KGN)}

The KGN has 80 permanent GPS sites that were established in 2000. Several governmental agencies including the Korea Astronomy and Space Science Institute (KASI) in the Ministry of Education, Science and Technology (MEST), the National Geographic Information Institute (NGI) in the Ministry of Land, Transport and Maritime Affairs (MLTM), the Ministry of Public Administration and Security (MOPAS), and other institutes have operated the GPS network and related facilities. Each reference station of the KGN is equipped with a geodetic dual frequency GPS receiver. The aims of the KGN are specified as the monitoring of local and regional crustal deformation, earth sciences research, cadastral surveys, providing geographic information, and transmitting Differential GPS (DGPS) corrections for a national service through some specific reference stations.

The KGN is distributed uniformly throughout the Korean Peninsula. The spatial resolution of the KGN is approximately $50 \mathrm{~km}$, which provides high spatial resolution observations. The geographical distribution of some of the permanent KGN GPS stations is shown in Fig. 1. For this study, thirty-eight GPS stations were selected in order to calculate local vertical TEC values and produce twodimensional TEC maps. They were chosen taking into ac-

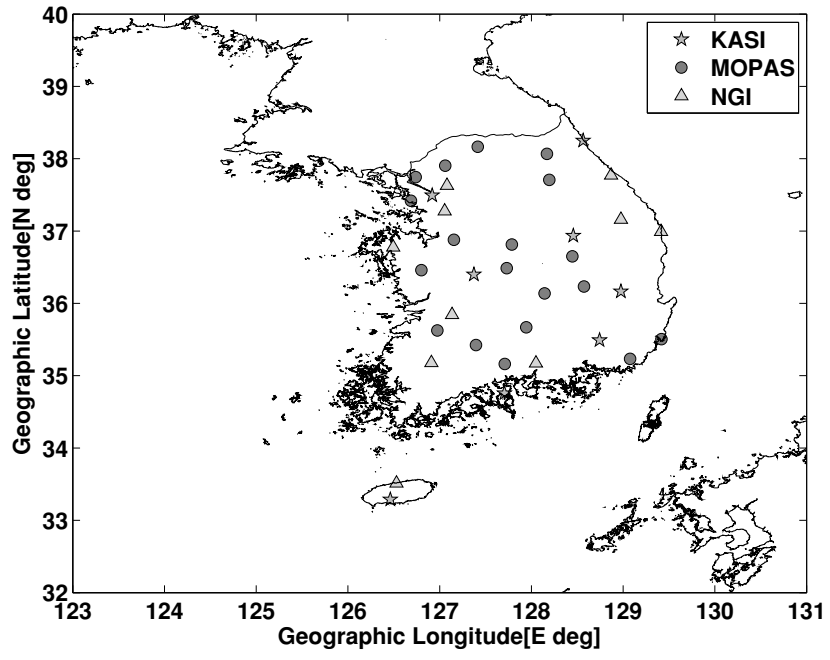

Fig. 1. The geographical distribution of GPS reference stations in South Korea. Reference stations of KASI are indicated as stars (green), while those of MOPAS and NGI are marked by circles (red) and triangles (cyan), respectively.

count their spatial distribution and the GPS data quality. A network of GPS stations can produce a spatially-distributed grid of TEC values. The receivers in GPS sites continuously provide data sampled every 30 seconds. This enables the production of grid-based GPS-TEC maps over South Korea with high temporal and spatial resolution.

\section{Method of Processing the GPS Data}

Dual-frequency GPS observations, including the carrier phase and code (pseudo-range) measurements, are combined to obtain ionospheric TECs. The ionosphere is a dispersive medium and both code and carrier phase measurements are affected by the ionosphere. Ionospheric TEC is quantified from GPS measurements by a linear combination of the code and carrier phase measurements from two carrier frequencies. The TEC is measured in TEC units with 1 TECU $\sim 10^{16}$ electrons $/ \mathrm{m}^{2}$. The fundamental equations for the code and carrier phase measurements can be expressed as follows (Schaer, 1999).

$$
\begin{aligned}
P= & \rho+c\left(\Delta t^{\mathrm{s}}-\Delta t^{\mathrm{r}}\right)+c\left(b^{\mathrm{s}}+b^{\mathrm{r}}\right) \\
& +\Delta_{\text {ion }}+\Delta_{\text {trop }}+\epsilon_{P} \\
\Phi= & \rho+c\left(\Delta t^{\mathrm{s}}-\Delta t^{\mathrm{r}}\right)-\Delta_{\text {ion }}+\Delta_{\text {trop }} \\
& +\lambda N+\epsilon_{\Phi}
\end{aligned}
$$

Where,

$\rho$ true geometric range from satellite to receiver (m)

$\Delta t^{\mathrm{s}} \quad$ satellite clock error (s)

$\Delta t^{\mathrm{r}} \quad$ receiver clock error (s)

$b^{\mathrm{s}} \quad$ satellite hardware bias (s)

$b^{\mathrm{r}} \quad$ receiver hardware bias (s)

$c \quad$ speed of light in a vacuum $(\mathrm{m} / \mathrm{s})$

$\Delta_{\text {ion }}$ ionospheric delay (m)

$\Delta_{\text {trop }}$ tropospheric delay (m)

$\lambda \quad$ wavelength of frequency (m)

$N \quad$ carrier phase integer ambiguity (cycle)

$\epsilon$ measurement noise (m) 
The estimated TEC values derived from the code measurements have a large uncertainty due to high noise levels. The carrier phase measurements are much more accurate than the code measurements, but carrier phase measurements require the resolution of phase ambiguity and consideration of infrequent cycle slips. To obtain a better accuracy for the derived GPS-TEC, a smoothing technique is employed with the code and carrier phase measurements. The smoothed code with the carrier phase is described as follows:

$P\left(t_{i}\right)_{\mathrm{sm}}=\omega_{i} P\left(t_{i}\right)+\left(1-\omega_{i}\right)\left[P\left(t_{i-1}\right)_{\mathrm{sm}}+\lambda \cdot\left(\Phi\left(t_{i}\right)-\Phi\left(t_{i-1}\right)\right)\right]$

Where $P\left(t_{i}\right)$ and $\Phi\left(t_{i}\right)$ are the pseudo-range and carrier phase observables: respectively. The term $\omega_{i}$ is the smoothing weight factor with an initial value of 1 and varying from 0 to 1 . The term $P\left(t_{i}\right)_{\mathrm{sm}}$ is the smoothed code.

The geometry-free linear combination of GPS observations is primarily used to obtain the ionospheric slant TEC (STEC).

$$
\begin{gathered}
\operatorname{STEC}_{P}=\left(\frac{1}{40.3}\right)[K]\left(P_{2}-P_{1}+B^{\mathrm{s}}+B_{\mathrm{r}}\right) \\
\mathrm{STEC}_{\Phi}=\left(\frac{1}{40.3}\right)[K]\left(L_{1}-L_{2}+\lambda_{1} N_{1}-\lambda_{2} N_{2}+B^{\mathrm{s}}+B_{\mathrm{r}}\right)
\end{gathered}
$$

Where $K=\left(f_{1}{ }^{2}{f_{2}}^{2}\right) /\left(f_{1}{ }^{2}-f_{2}{ }^{2}\right), B^{\mathrm{s}}=b_{2}{ }^{\mathrm{s}}-b_{1}{ }^{\mathrm{s}}$ and $B_{\mathrm{r}}=b_{2 \mathrm{r}}-b_{1 \mathrm{r}}$ represent the differential code biases (DCBs). When estimating the ionospheric TEC using GPS data, the GPS-DCBs have to be taken into account. If the DCBs are not removed from the TEC value, the "absolute" TEC value cannot be determined. Moreover, GPS receiver DCBs can be as much as a few tens of nanoseconds (ns). The DCBs for all GPS satellites and ground stations are computed daily as constants. In our approach, the estimation of the receiver DCB needs one particular value as reference in the GPS network. We also used a zero-mean condition for the separation of the receiver-dependent part of the bias. The DCB needs to be removed from the original TEC as it results in a decrease of reliability of the ionospheric TEC.

To obtain normalized data, the STEC value is converted to an equivalent vertical TEC (VTEC) value at an ionosphere pierce point (IPP). Therefore, the slant TEC is multiplied by the standard geometric mapping function, which, in general, is de ned as:

$$
\begin{gathered}
\operatorname{VTEC}=\frac{\operatorname{STEC}}{\operatorname{MF}(E)} \\
\operatorname{MF}(E)=\left[\frac{1}{\sqrt{\left(1-\left(R_{\mathrm{E}} \cdot \cos (E) /\left(R_{\mathrm{E}}+h_{\mathrm{IPP}}\right)^{2}\right)\right.}}\right]
\end{gathered}
$$

Where, $E$ is the elevation angle of the GPS satellite; $R_{\mathrm{E}}$ is the Earth's radius $(6378.137 \mathrm{~km})$, and $h_{\mathrm{IPP}}$ is the height of the assumed ionosphere thin shell. Here, the height $h_{\text {IPP }}$ is taken to be $350 \mathrm{~km}$. Recent studies have referred improvement level in accuracy of mapping TEC compared with the thin shell approach. For a given GPS reference station location $\left(\lambda_{r}, \phi_{r}\right)$, satellite azimuth and elevation angle $\left(A_{z}, E\right)$ and assumed ionosphere thin shell height, the geographic latitude $\left(\phi_{\text {IPP }}\right)$ and longitude $\left(\lambda_{\text {IPP }}\right)$ of an IPP are calculated as follows:

$$
\begin{array}{r}
\psi=\frac{\pi}{2}-E-\sin ^{-1}\left[\left(\frac{R_{\mathrm{E}}}{R_{\mathrm{E}}+h_{\mathrm{IPP}}}\right) \cdot \cos (E)\right] \\
\theta_{\mathrm{IPP}}=\sin ^{-1}\left[\sin \phi_{r} \cos \psi+\cos \phi_{r} \sin \psi \cos \left(A_{z}\right)\right] \\
\lambda_{\mathrm{IPP}}=\lambda+\sin ^{-1}\left[\frac{\sin \psi \cdot \sin \left(A_{z}\right)}{\cos \phi_{\mathrm{IPP}}}\right]
\end{array}
$$

Where $\psi$ is the subtended angle between the satellite and station position. The inverse distance weighted (IDW) method is an interpolation method (Lancaster and Salkauskas, 1986) which uses known values at certain distributed points to predict the values at the grid points. This method is based on the assumption that the interpolation is in uenced most by the nearest points and less by more distant points. A general form of interpolating a value using IDW is as follows:

$$
Z_{0}=\frac{\sum_{i=1}^{S} Z_{i} \cdot\left(\frac{1}{d_{i}^{k}}\right)}{\sum_{i=1}^{S}\left(\frac{1}{d_{i}^{k}}\right)}
$$

Where $Z_{0}$ is the value of an interpolated grid point; $Z_{i}$ is a known value, $d_{i}$ is the distance between a point being estimated and a samplied point, $k$ is an exponent parameter, and $S$ is the total number of known points. We applied the IDW method to establish a GPS grid-based ionospheric model over South Korea. The ionospheric TEC maps were produced using a $0.1^{\circ}$ grid spacing. To reduce the effects of ray-path bending of GPS signals, the receiver's elevation mask was set to 15 degrees.

\section{Results}

\subsection{A typical diurnal pattern of TEC variation in} November 2003

We have developed a regional ionosphere model (RIM) using data from the KGN. The RIM has a spatial resolution of $0.1^{\circ} \times 0.1^{\circ}$ at a region located between $32^{\circ}-40^{\circ}$ geographic latitude $(\mathrm{N})$ and $123^{\circ}-131^{\circ}$ longitude $(\mathrm{E})$ and a time resolution of 30 seconds. To analyze the normalized characteristics of the vertical TEC variation over South Korea, we processed GPS data for one month. Some of the results are shown in Fig. 2 which presents an example of the TEC variation at a certain point $\left(37^{\circ} \mathrm{N}, 127^{\circ} \mathrm{E}\right)$ during 10 geomagnetically quiet days with $K_{\mathrm{p}}$ less than 4 in November 2003.

The temporal variation of TEC at any point for the 10 quiet days represents the general trends. The diurnal pattern of vertical TEC exhibits a gradual increase from about early morning (UT $\sim 0 \mathrm{~h}$ ) to an afternoon maximum (UT $=5 \sim$ $6 \mathrm{~h}$ ) and then decreases sharply until about sunset (UT $11 \mathrm{~h})$. The daily peak occurred around 6:00 UT (UT $=\mathrm{LT}-$ 09:00). These patterns of TEC variation help appreciate its day-to-day variability. The median diurnal TEC variation for this period is determined for the purpose of making a comparison with TEC variations monitored during storm times. 


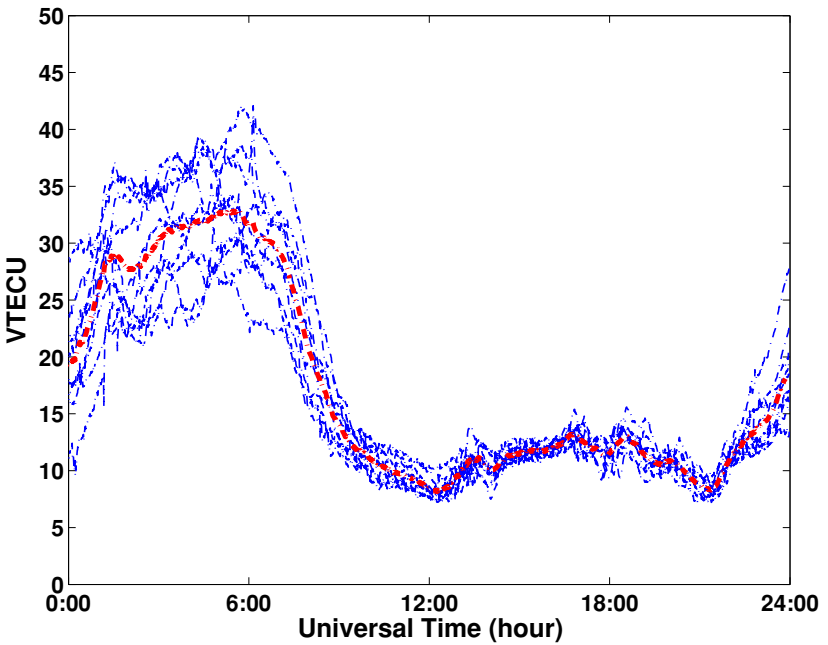

Fig. 2. The diurnal pattern of vertical TEC variation for 10 quiet days in November 2003. The red dotted line represents the median TEC variation at any point for this period.

\subsection{Ionospheric TEC responses to geomagnetic storms}

Two large geomagnetic events occurred on 4 and 20 November 2003. We investigated the ionospheric TEC responses at each point of the mid-latitude network during these events.

4.2.1 4 November, 2003 We used GPS data from the KGN to monitor the ionospheric TEC response over South Korea when a geomagnetic storm occurred on 4 November, 2003. At this time, an energetic solar eruption, named the X28 flare, was observed. Fortunately, this flare did not influence the region near the Earth. The geomagnetic storm occurred at daytime locally. To quantify the geomagnetic activity, Fig. 3 shows the variations of three geomagnetic activity indices and the auroral electroject $\left(A_{\mathrm{E}}\right)$ index for 3-5 November 2003. The top image in Fig. 3 shows the variation in the $A_{\mathrm{E}}$ index. The first and second peaks of the $A_{\mathrm{E}}$ index were observed at about 07:00 and 10:30 UT, respectively, on 4 November. The maximum $A_{\mathrm{E}}$ index value is $2011 \mathrm{nT}$ (nano Tesla), at the second peak. The variation of the $K_{\mathrm{p}}$ index is presented in the middle image of Fig. 3. The three-hourly $K_{\mathrm{p}}$ index reached its maximum value when the second peak of the $A_{\mathrm{E}}$ index took place at 10:30 UT. The bottom image in Fig. 3 presents the variation of the disturbance storm time $\left(D_{\mathrm{st}}\right)$ value. The intensity of the geomagnetic storm is commonly defined by the minimum $D_{\text {st }}$ value. The minimum hourly $D_{\text {st }}$ value during the geomagnetic storm was $-69 \mathrm{nT}$ at 10:30 UT. We can see that there is a close correlation among these variations in the geomagnetic activity indices and the $A_{\mathrm{E}}$ index. The geomagnetic data are available through the website http://swdcwww.kugi.kyoto-u.ac.jp/wdc/Sec3.html.

To investigate the characteristics of the ionospheric TEC responses during this period, the ionospheric TEC was derived from the GPS data collected from the KGN in South Korea. The two-dimensional TEC maps of the KASI-RIM have a $0.1^{\circ}$ grid spacing and a very high time resolution of 30 seconds.

Figure 4 presents the diurnal vertical TEC variation at a particular grid point $\left(37^{\circ} \mathrm{N}, 127^{\circ} \mathrm{E}\right)$ over South Korea
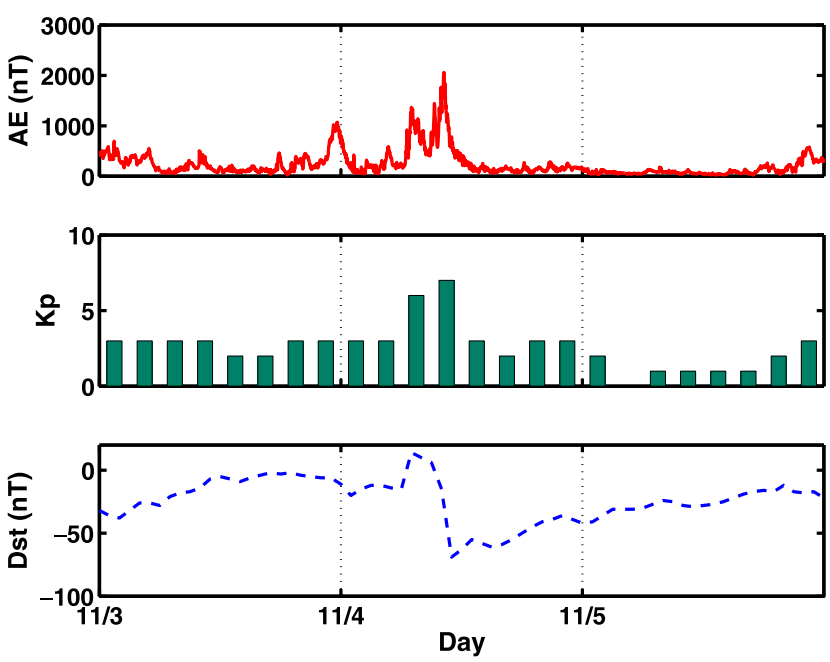

Fig. 3. Time series of the $A_{\mathrm{E}}$ index (red solid line on the upper image), and the $K_{\mathrm{p}}$ (green boxes on the middle image) and $D_{\text {st }}$ (blue dotted line on the lower image) geomagnetic activity indices on 3-5 November 2003 observed at the Kakioka Geomagnetic Observatory in Japan.

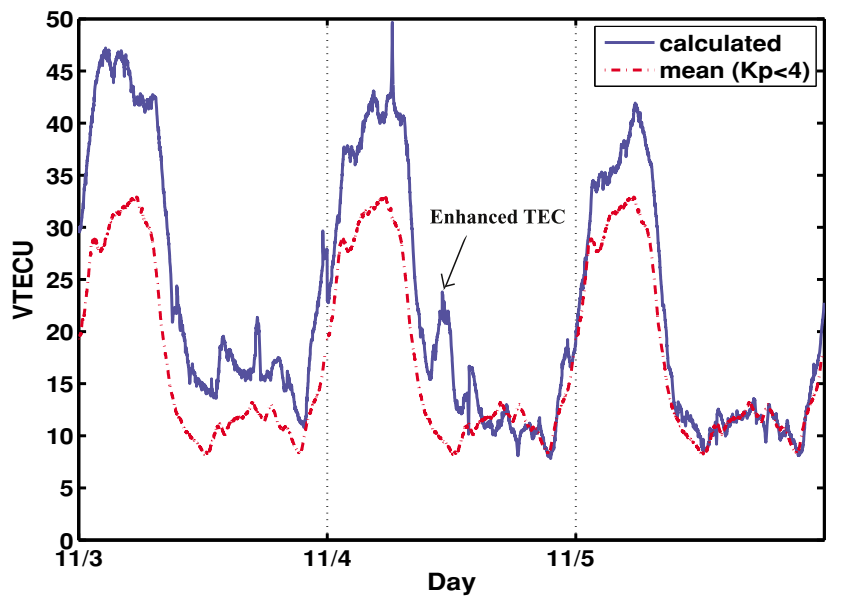

Fig. 4. The diurnal vertical TEC variation at a specific point $\left(37^{\circ} \mathrm{N}\right.$, $127^{\circ} \mathrm{E}$ ) over South Korea on 3-5 November 2003 using KGN data. The blue line represents the vertical TECU obtained by the KASI regional ionosphere model. The red dotted line is the diurnal median TEC variation obtained for 10 quiet days $\left(K_{\mathrm{p}}<4\right)$.

from 3 to 5 November, 2003. The output TEC is produced every 30 seconds. During this period of the geomagnetic storm, the variation in GPS-TEC was from 10 to 50 vertical TECU at the given point. In these disturbed conditions, the instantaneous enhancement of the TEC can be observed in Fig. 4. The relative amplitude level of the TEC increased by about 10 TECU at 10:30 UT on 4 November 2003. This sharp rise in TEC is more obvious when comparing with the calculated TEC at the same time on 5 November.

The variations in the GPS-TEC show similar fluctuations to the variations of the geomagnetic indices. The purpose of Figs. 3 and 4 are to establish the correlation between variations in the GPS-TEC and geomagnetic storms at midlatitudes, especially over South Korea. To illustrate the typical diurnal variation over South Korea, the diurnal median TEC obtained for a quiet period is also presented in Fig. 4. Figure 5 shows the two-dimensional TEC map as a function 


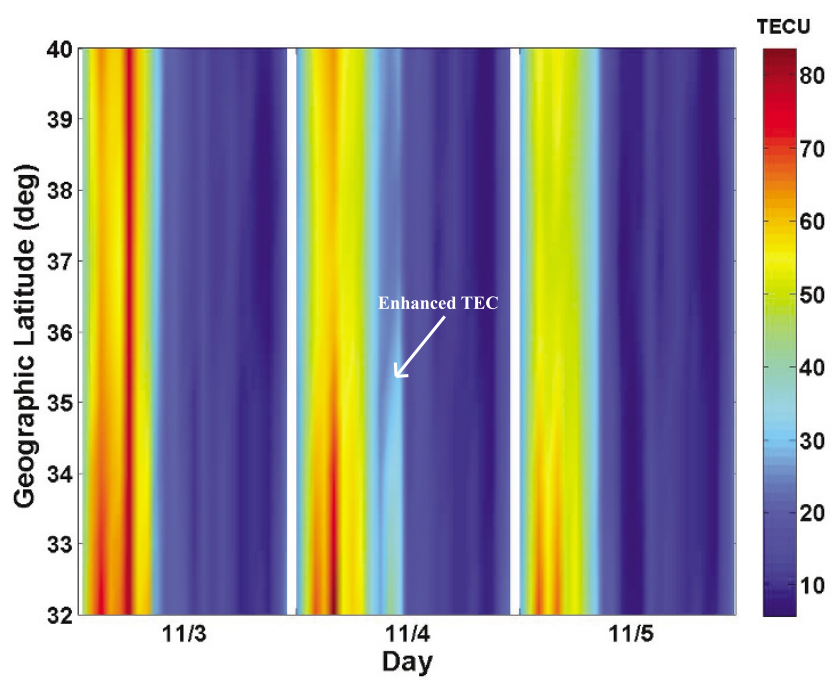

Fig. 5. Two-dimensional TEC distributions as a function of geographic latitude ( $Y$-axis) and time ( $X$-axis) for 3-5 November, 2003. The full range of the geographic latitude is from $32^{\circ}$ to $40^{\circ} \mathrm{N}$.
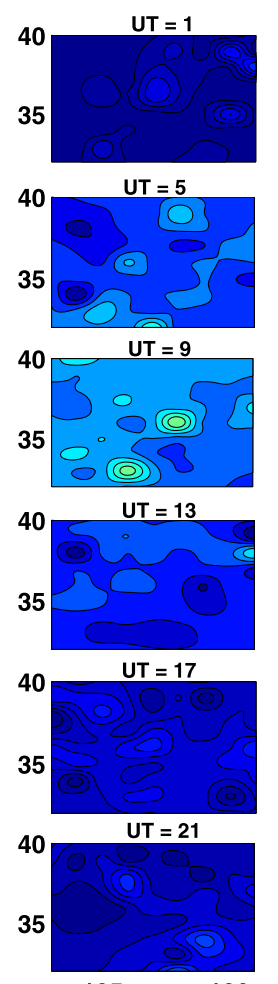

125

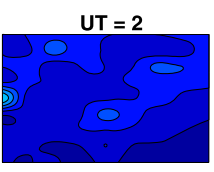

UT $=6$

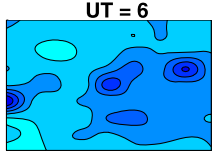

UT $=10$

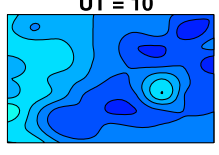

UT $=14$

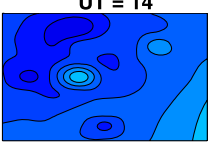

UT $=18$

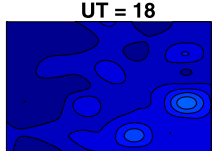

UT $=22$

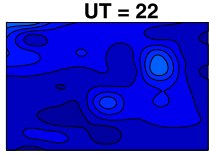

$125 \quad 130$

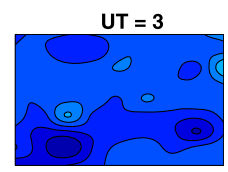

UT $=7$

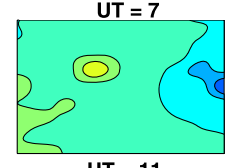

UT $=11$
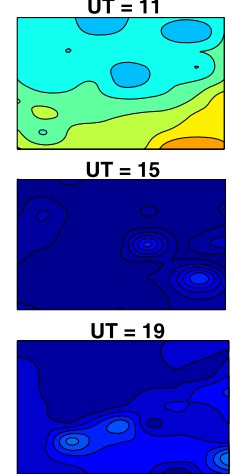

UT $=23$

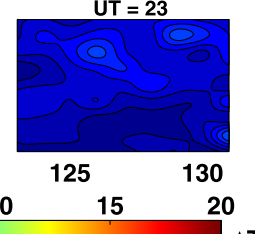

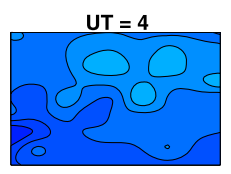

$\mathrm{UT}=8$

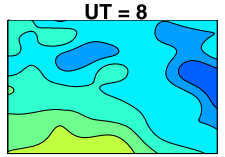

UT $=12$

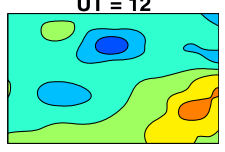

UT $=16$
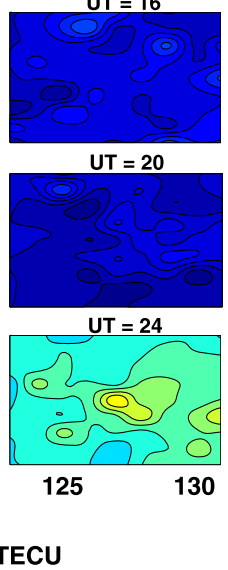

Fig. 6. Snap-shots of the TEC differences between a disturbed day (4 November 2003) and a quiet day (5 November 2003). The $x$-axis is the geographic longitude and the $y$-axis is the geographic latitude for all of the images.

of time and geographic latitude. This TEC map provides more insight into the actual disturbances during a geomagnetic storm. In particular, the GPS-TEC map reveals an enhanced TECU during 10:30 12:00 UT. The effects of the daytime storm on 4 November 2003 shown in Figs. 3 and 4 can be compared with the TEC behavior shown in Fig. 5. This is associated with irregular geomagnetic activity. It is evident that the mid-latitude ionosphere was affected by the geomagnetic storm that occurred on 4 November.
To clearly identify the ionospheric changes during the storm, the storm-time TEC relative to the TEC for quiet conditions was computed. Figure 6 shows snap-shots of the two-dimensional TEC differences between the storm day of 4 November and the quiet day of 5 November 2003. From this 2-D TEC map, there were large differences in TEC values at $\mathrm{UT} \sim 11: 00$ and $\mathrm{UT} \sim 12: 00$. This result agrees well with the previous results presented in Figs. 4 and 5. 

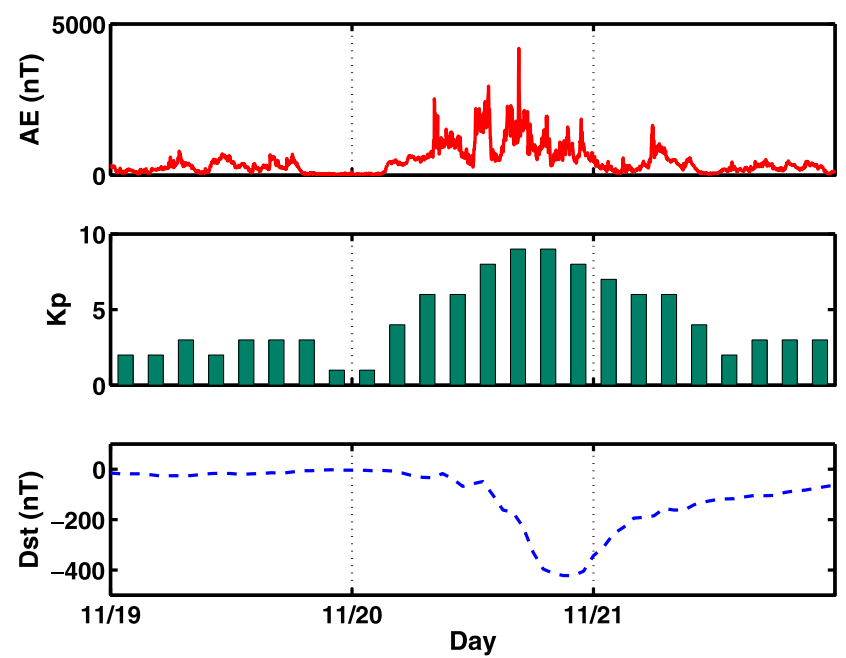

Fig. 7. Time series of the $A_{\mathrm{E}}$ index (red solid line on the upper image), and the $K_{\mathrm{p}}$ (green boxes on the middle image) and $D_{\mathrm{st}}$ (blue dotted line on the lower image) geomagnetic activity indices during the period 19-21 November 2003.

4.2.2 20 November, 2003 Gopalswamy et al. (2005) investigated the largest geomagnetic storm that occurred on 20 November 2003, due to a coronal mass ejection (CME) from the Sun. Figure 7 shows the variations of the $A_{\mathrm{E}}$ index and geomagnetic activity indices. The SSC occurred at around 08:30 UT on 20 November 2003. During this storm, the geomagnetic activity $K_{\mathrm{p}}$ index reached extreme values of $\sum K_{\mathrm{p}}=50+$. The three-hourly $K_{\mathrm{p}}$ index indicates that intense activity occurred after 06:00 UT, reaching values over $6+$. The minimum $D_{\mathrm{st}},-422 \mathrm{nT}$, was recorded at 21:00 UT. The maximum $K_{\mathrm{p}}$ and $A_{\mathrm{E}}$ indices were $9-$ and $4,192 \mathrm{nT}$, respectively. Sharp increases in the $A_{\mathrm{E}}$ index were observed at about 08:00 UT, 12:00 UT and 16:30 UT on this day. The auroral activity was very high at 16:00 UT.

The results obtained from the KGN on 19-21 November 2003 and presented in Fig. 8 shows the diurnal vertical TECU variation at a particular point $\left(37^{\circ} \mathrm{N}, 127^{\circ} \mathrm{E}\right)$ over South Korea. The maximum TECU value occurs at around 5:00 UT. During this period sharp increases of the TEC occurred at about 11:00 UT and 23:00 UT as shown in Fig. 8. In Fig. 7, the first response of the ionospheric TEC derived from the GPS network was at about 11:00 UT. The TEC was enhanced by about 10 TECU at all grid points within a short time. Prominent increases or decreases of TEC were seen beyond 13:00 UT. A steady TEC increase was observed from 11:00 UT to 13:00 UT as shown in Fig. 8. The largest TEC enhancement occurred at 13:00 UT. In contrast, a sudden TEC decrease took place simultaneously after the TEC peak. This decrease is sometimes anticipated due to the rapid TEC enhancement. The rapid fluctuations of GPS-TEC from 11:00 UT to 13:00 UT correspond with the variations in the $K_{\mathrm{p}}$ and $A_{\mathrm{E}}$ indices during this time. The periods of variation in the geomagnetic indices in Fig. 7 were coincidental with the periods of GPS-TEC enhancement shown in Fig. 8. The geomagnetic and auroral activities reached their maximums at 16:30 UT during this storm, but a sudden enhancement of the GPS-TEC was not apparent at the same time. This GPS-TEC signature also does not

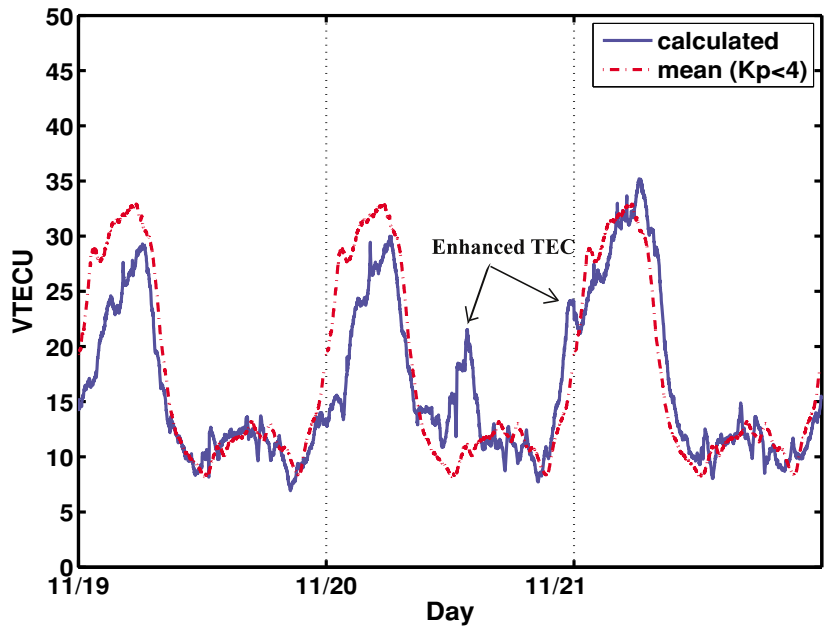

Fig. 8. The diurnal vertical TEC variations at a specific point $\left(37^{\circ} \mathrm{N}\right.$, $127^{\circ}$ E) over South Korea on 19-21 November 2003 using KGN data. The blue line represents the vertical TECU obtained by the KASI regional ionosphere model. The red dotted line is the diurnal median TEC variation obtained for 10 quiet days $\left(K_{\mathrm{p}}<4\right)$.

appear in Fig. 9, which depicts a two-dimensional map of vertical TECs as a function of geographic latitude and time.

Figure 10 shows the difference in the two-dimensional TEC between the geomagnetically disturbed day (on 20 November) and the quiet day (on 19 November). From this, it can be seen that the TEC gradually increased starting at 10:00 UT. The first TEC enhancement reached its maximum around 13:00 14:00 UT and increased rapidly by 22 TEC units in two hours. The second TEC enhancement started at about 23:00 UT with a magnitude which was larger than that of the first one.

Overall, the analyses showed that the geomagnetic activity indices and the GPS-TEC responses obtained from the $\mathrm{KGN}$ were in good agreement at the SSCs that occurred on 4 and 20 November 2003. However, the TEC increments for all the events that occurred at these mid-latitudes were not always proportional to the geomagnetic activity indices. Also, unfortunately, we could not present results for a comparison of GPS-derived TEC and ionosonde TEC due to the loss of some of the ionosonde data at the Anyang station $\left(37.39^{\circ} \mathrm{N}, 126.95^{\circ} \mathrm{E}\right)$.

\section{Conclusions}

The ionosphere is easily affected by environmental factors. Variations in ionospheric TEC can be detected by local observation data. This paper has examined the ionospheric response over South Korea during the geomagnetic storms on 4 and 20 November 2003 using the permanent Korean GPS network. This study has investigated the similarities between the geomagnetic storms and the local TEC responses observed by a GPS ionospheric model.

In this paper, the grid-based TEC values with high temporal and spatial resolutions were obtained using an inverse distance weighted (IDW) interpolation method. This enables observations of a fast TEC response to variations in conditions, due to the high temporal resolution of $30 \mathrm{sec}-$ onds. To clearly identify the ionospheric TEC changes that occur during storm periods, we have presented the diurnal 


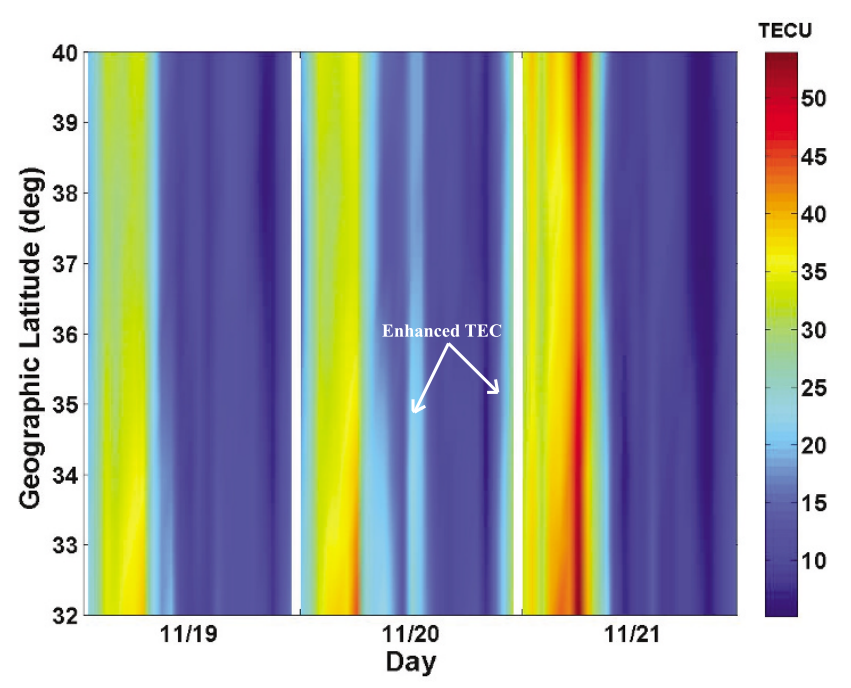

Fig. 9. Two-dimensional TEC distributions as a function of geographic latitude ( $Y$-axis) and time ( $X$-axis) for 19-21 November, 2003. The full range of the geographic latitude is from $32^{\circ}$ to $40^{\circ} \mathrm{N}$.
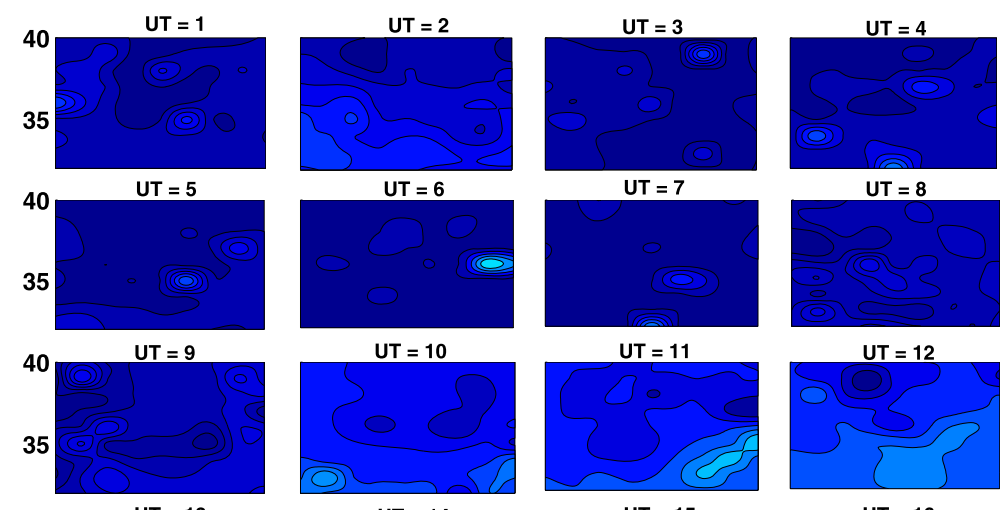

UT $=11$

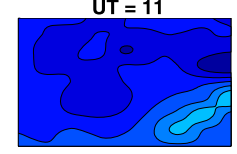

UT $=12$

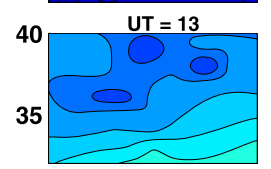

$\mathrm{UT}=14$

$\mathrm{UT}=15$
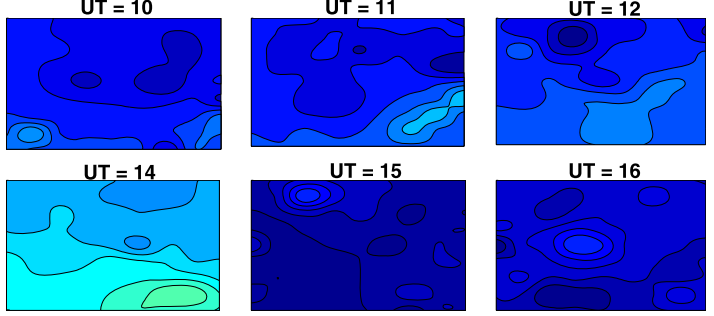

$\mathrm{UT}=\mathbf{1 6}$
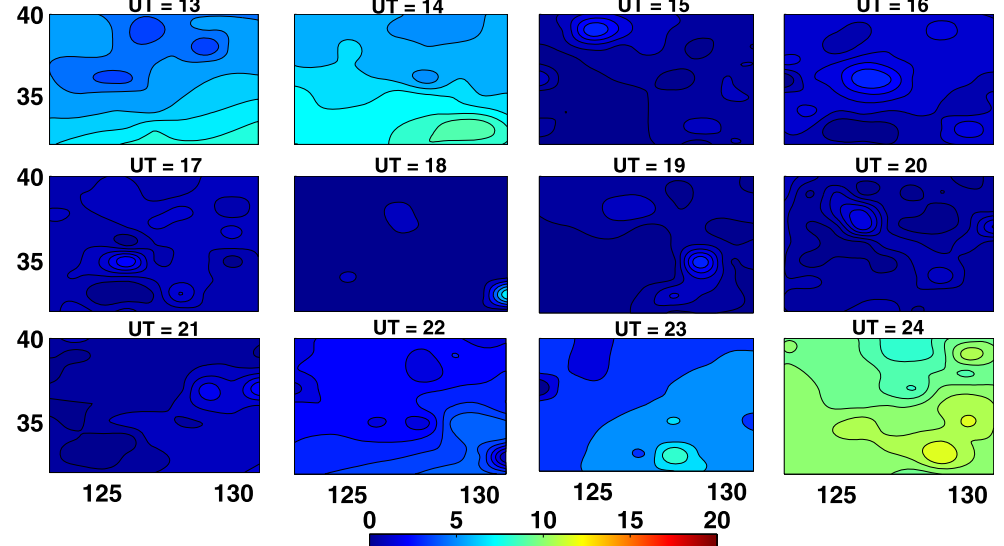

$\triangle T E C U$

Fig. 10. Snap shots of the TEC differences between a disturbed day (20 November 2003) and a quiet day (19 November 2003 ). The $x$-axis is the geographic longitude and the $y$-axis is the geographic latitude for all of the images.

pattern of TEC variation over the Korean Peninsula for quiet conditions as well.

We have investigated the GPS-TEC variations of the ionosphere on 4 November and 20 November 2003. The sudden changes in GPS-TEC observed during these storm periods were remarkable. An enhancement of GPS-TEC occurred almost simultaneously with the timing of the sudden storm commencements (SSCs). It has been shown that the small-scale TEC responses derived from this GPS network were closely related with geomagnetic activities. However, the GPS-TEC responses for all of the storm events were not proportional to the geomagnetic activity. The study of the correlation between the TEC response and the geomagnetic activity at mid-latitudes requires further investigation.

Our approach, using high temporal and spatial resolution measurements of TEC, will contribute to the monitoring of ionospheric TEC at mid-latitudes and the study of geomag- 
netic storms. As a result, GPS can be used as a comprehensive tool for the study of geomagnetic storms and verication of other measurements. Therefore, we believe that the changes in ionospheric composition generated by such storms are the cause of VTEC enhancements observed in the mid-latitudes.

Acknowledgments. The authors appreciate the referee's comments which were helpful in improving our manuscript. The authors also would like to thank the data centers, the National Geographic Information Institute (NGI) in the Ministry of Land, Transport and Maritime Affairs (MLTM), the Ministry of Public Administration and Security (MOPAS) and the Data Analysis Center for Geomagnetism and Space Magnetism Graduate School of Science, Kyoto University for providing us with the GPS data and the geomagnetic indices data. This work was supported by the KASI's basic core technology.

\section{References}

Davis, K. and G. K. Hartmann, Studying the ionosphere with the Global Positioning System, Radio Sci., 32, 1695-1703, 1997.

Fedrizzi, M., E. de Paula, I. J. Kantor, R. Langley, A. Komjathy, and I. Batista, Study of the March 31, 2001 magnetic storm effects on the ionospheric GPS data, Adv. Space Res., 36, 534-545, 2005.

Gopalaswamy, N., S. Yashiro, G. Michalek, H. Xie, R. P. Lepping, and R. A. Howard, Solar source of the largest geomagnetic storm of cycle 23, Geophys. Res. Lett., 32, 12-34, 2005.

Hernandez-Pajares, M., J. M. Juan, and J. Sanz, New approaches in global ionospheric determination using ground GPS data, J. Atmos. Terr. Phys., 61, 1237-1247, 1999.

Immel, T. J., S. B. Mende, H. U. Frey, N. Ostgaard, and G. R. Gladstone, Effect of the 14 July 2000 solar are on Earth's FUV emissions, $J$. Geophys. Res., 108, 1155, 2003.

Jakowski, N., S. Heise, S. Wehrenpfenning, S. Schluter, and R. Reimer, GPS/GLONASS-based TEC measurements as a contributor for space weather forecast, J. Atmos. Sol. Terr. Phys., 64, 729-735, 2002.

Jin, S. G., J. Wang, H. P. Zhang, and W. Y Zhu, Real-time monitoring and prediction of Ionosphere Electron Content by means of GPS, Chinese Astron. Astrophys., 28, 331-337, 2004.

Klobuchar, J. A., Ionospheric time-delay algorithm for single-frequency GPS users, IEEE Transactions on Aerospace and Electronic Systems, 23, 325-331, 1987.

Lancaster, P. and K. Salkauskas, Curve and Surface Fitting: An Introduction, Academic Press, London Orlando, 1986.

Lanyi, G. E. and T. Roth, A comparison of mapped and measured total ionospheric electron content using Global Positioning System and beacon satellite observations, Radio Sci., 23, 483-492, 1988.
Lei, J., L. Liu, W. Wan, and S. R. Zang, Modeling investigation of ionospheric storm effects over Millstone Hill during August 4-5, 1992, Earth Planets Space, 56, 903-908, 2004.

Liao, X., Carrier phase based ionosphere recovery over a regional area GPS network, Rep. UCGE, 120, University of Calgary, Calgary, Canada, 2000.

Mannucci, A., B. Wilson, and C. Edwards, A new method for monitoring the Earth's ionospheric total electron content using the GPS global network, in Proceedings of ION GPS'93, pp. 22-24, Inst. of Navig., Alexsandria, 1993.

Mannucci, A. J., B. T. Tsurutani, B. A. Iijima, A. Komjathy, A. Saito, W. D. Gronzalez, F. L. Guarnieri, J. U. Kozyra, and R. Skone, Dayside global ionospheric response to the major interplanetary events of October 29-30 "Halloween Storms", Geophys. Res. Lett., 32, L12S02, doi:10.1029/2004GL021467, 2005.

Otsuka, Y., T. Ogawa, A. Saito, and T. Tsugawa, A new technique for mapping of total electron content using GPS network in Japan, Earth Planets Space, 54, 63-70, 2002.

Sardon, E., A. Rius, and N. Zarraoa, Estimation of the transmitter and receiver differential biases and the ionospheric total electron content from Global Positioning System observations, Radio Sci., 29, 577-586, 1994.

Schaer, S., Mapping and predicting the Earth's ionosphere using the Global Positioning System, Ph.D. thesis, University of Berne, Switzerland, 205 pp., 1999.

Skone, S., Wide area ionosphere grid modeling in the auroral region, Ph.D thesis, University of Calgary, Canada, 198 pp., 1998.

Tsugawa, T., N. Kotake, Y. Otsuka, and A. Saito, Medium-scale traveling ionospheric disturbance observed by GPS receiver network in Japan: a short review, GPS Solution, 11, 139-144, 2006.

Tsurutani, B. T., A. J. Mannucci, B. A. Iijima, J. H. Sobral, W. D. Gonzalez, F. L. Gaurnieri, T. Tsuda, A. Saito, K. Yumoto, B. Fejer, T. FullerRowell, J. U. Kozyra, J. C. Foster, A. J. Coster, and V. M. Vasyliunas, Global dayside ionospheric uplift and enhancement associated with interplanetary electric elds, J. Geophys. Res., 109, A08302, 2004.

Tsurutani, B. T., A. J. Mannucci, B. A. Iijima, F. L. Guarnieri, W. D. Gonzalez, D. L. Judge, P. Gangopadhyay, and J. Pap, The extreme Halloween 2003 solar ares (and Bastille Day, 2000 Flare), ICMEs, and resultant extreme ionospheric effects: A review, Adv. Space Res., 37, 1583-1588, 2006.

Wan, W., L. Liu, H. Yuan, B. Ning, and S. Zhang, The GPS measured SITEC caused by the very intense solar are on July 14, 2000, Adv. Space Res., 36, 2465-2469, 2005.

Zhao, B., W. Wan, and L. Liu, Responses of equatorial anomaly to the October-November 2003 superstorms, Ann. Geophys., 23, 693-706, 2005 .

B.-K. Choi (e-mail: bkchoi@kasi.re.kr), S.-J. Lee, and J.-U. Park 